\title{
SUR UN THÉORÈME DE M. MATSUSHIMA
}

\author{
KENTARO YANO
}

§. Dans une Note parue dans ce Journal, M. Matsushima [1] a démontré le

THÉoRÈme. Dans un espace compact de Kähler-Einstein dont la courbure est positive, un vecteur analytique contrevariant u peut s'écrire d'une et d'une seule manière sous la forme $u=v+F w$ où $v$ et $w$ sont tous les deux les vecteurs de Killing et $F$ le tenseur définissant la structure complexe de l'espace.

Dans cette Note on va donner deux démonstrations de ce théorème en utilisant la condition nécessaire et suffisante pour qu'un vecteur soit analytique contrevariant. La première est donnée dans $\S 3$ et la deuxième dans $\S 4$.

$\S 1$. Considérons un espace $V$ de Riemann compact et orientable de $n$ dimensions dont la métrique $d s^{2}=g_{j i}(\xi) d \xi^{j} d \xi^{i}$ est définie positive. On désigne par $d$ l'opérateur qui opère à un tenseur symétrique gauche $u: u_{i_{1}, i_{p-1} \ldots l_{1}}$ de degré $p$ et donne un tenseur symétrique gauche de degré $p+1, d u: \nabla_{i} \imath t_{i_{1}, i_{r^{\prime}-1} \ldots i_{1}}$ $-\nabla_{i_{p}} u_{i i_{p-1} \ldots i_{1}}-\ldots-\nabla_{i_{1}} u_{i_{p} \ldots i_{2} i}$ et par $\delta$ l'opérateur qui donne un tenseur symétrique gauche de degré $p-1, \delta u:-g^{j i} \nabla_{j} u_{i i_{p_{1-1}} \ldots i_{1}}, \nabla_{j}$ étant l'opérateur de la dérivée covariante par rapport aux symboles de Christoffel $\left\{\begin{array}{l}h \\ j i\end{array}\right\}$. Il est bien connu que $d d u=0$ et $\delta \delta u=0$.

On désigne par $\Delta$ l'opérateur $\delta a+d \delta$. Pour un vecteur $u$, on a $\Delta u$ : $-\left(g^{j i} \nabla_{j} \nabla_{l} u_{h}-K_{h}{ }^{i} u_{i}\right), K_{h}{ }^{i}$ étant le tenseur de Ricci. On peut facilement vérifier que $\Delta d u=d \Delta u$ et $\Delta \delta u=\delta \Delta u$.

On définit le produit global de deux tenseurs symétriques gauches

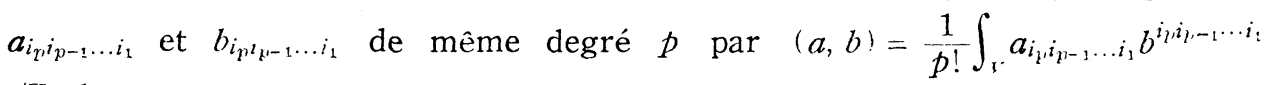
$\sqrt{g} d \xi^{1} \wedge \ldots \wedge d \xi^{n}, g$ étant le déterminant formé avec $g_{j i}$.

Il est bien connu que $(d u, v)=(u, \delta v),(\delta u, v)=(u, d v)$ et $(\Delta u, u)$ $=(d u, d u)+(\delta u, \delta u)$. La dernière équation démontre un théorème célèbre: Pour qu'un tenseur symétrique gauche soit harmonique, il faut et il suffit que $\Delta u=-0$.

Received May 25, 1957. 
Exactement de la même manière que la précédente, on désigne par $D$ l'opérateur qui opère à un tenseur symétrique $u: u_{i_{\nu}, i_{p-1} \ldots i_{1}}$ de degré $p$ et donne un tenseur symétrique de degré $p+1, D u: \nabla_{i} u_{i_{\nu}, i_{\eta^{-1}} \ldots i_{*}}+\nabla_{i_{\nu}} u_{i i_{1}-1 \ldots i_{1}}+\ldots$ $+\nabla_{i_{1}} u_{i_{\mu}, i_{1}-1 \ldots i_{2} i}$, et par $\delta$ l'opérateur qui donne un tenseur symétrique de degré $p-1, \delta u:-g^{j i} \nabla_{j} u_{i i_{1},-1} \ldots i_{1}$. Si l'on pose $\square=\delta D-D \delta$, on a, pour un vecteur $u$, $\square u:-\left(g^{j i} \nabla_{j} \nabla_{i} u^{h}+K_{i}^{h} u^{i}\right)$.

Si l'on définit le produit global de deux tenseurs symétriques $a_{i_{v} i_{p-1} \ldots i_{1}}$ et $b_{i_{p} i_{v-1} \ldots i_{1}}$ par la même formule que la précédente, on trouve $(D u, v)=(u, \delta v)$, $(\delta u, v)=(u, D v)$ et $(\square u, u)=(D u, D u)-(\delta u, \delta u)$. Un vecteur de Killing $u$ est caractérisé par $D u=0$ (et $\delta u=0$ ) et par conséquent satisfait à $\square u=0$. La dernière équation démontre le

THÉók̀me. Pour qu'un vecteur $u$ soit un vecteur de Killing, il faut et il suffit que $\square u=0$ et $\delta u=0$.

§. Considérons un espace compact de Kähler et désignons par $F$ le tenseur qui définit la structure complexe de l'espace. Alors on a $F_{j}^{i} F_{i}^{h}=-A_{j}^{h}$, $F_{j i}=-F_{i j}$ et $\nabla_{j} F_{i h}=0$.

Un tenseur $u_{j i}$ est dit pur s'il satisfait à ${ }^{*} O u=0: \frac{1}{2}\left(A_{j}^{c} A_{i}^{b}+F_{j}^{c} F_{i}^{b}\right) u_{c b}=0$ et hybride s'il satisfait à $O u=0: \frac{1}{2}\left(A_{j}^{c} A_{i}^{b}-F_{j}^{c} F_{i}^{b}\right) u_{c b}=0$. De même un tenseur $u_{i}^{h}$ est dit pur s'il satisfait à ${ }^{*} O u=0: \frac{1}{2}\left(A_{i}^{b} A_{a}^{h}+F_{i}^{b} F_{a}{ }^{h}\right) u_{b}{ }^{a}=0$ et hybride s'il satisfait à $O u=0: \frac{1}{2}\left(A_{i}^{b} A_{a}^{h}-F_{i}^{b} F_{a}{ }^{h}\right) u_{b}{ }^{a}=0$. Il est facil de voir que les opérateurs $O$ et ${ }^{*} O$ satisfont aux $O+{ }^{*} O=A, O \cdot O=0, O \cdot{ }^{*} O={ }^{*} O \cdot O=0,{ }^{*} O \cdot{ }^{*} O$ $=* O, A$ étant l'opérateur identique.

Or un vecteur $u_{i}$ est dit analytique covariant si sa dérivée covariante $\nabla_{j} u_{i}$ est pure, c'est-à-dire, si l'on a ${ }^{*} O \nabla u=0: \frac{1}{2}\left(A_{j}^{c} A_{i}^{b}+F_{j}^{c} F_{i}^{b}\right) \nabla_{c} u_{b}=0 . \quad$ Entre les opérateurs $\Delta$ et $* O V$ appliqués à un vecteur covariant, on a les relations $2 \Delta u$ $=-\delta^{*} O \nabla u$ et $(\Delta u, u)=\left({ }^{*} O \nabla u,{ }^{*} O \nabla u\right)$, qui démontrent le

THÉoRÈme. Pour qu'un vecteur soit analytique covariant, il faut et il suffit que $\Delta u=0$, c'est-à-dire, que le vecteur soit harmonique.

Un vecteur $u^{h}$ est dit analytique contrevariant, si sa dérivée covariante $\nabla_{j} u^{h}$ est pure, c'est-à-dire, si l'on a $* O \nabla u=0: \frac{1}{2}\left(A_{i}^{b} A_{a}^{h}+F_{i}^{b} F_{a}{ }^{h}\right) \nabla_{b} u^{a}=0$. Entre les operateurs $\square$ et $* O \nabla$ appliqués à un vecteur contrevariant, on a les relations $2 \sim u=-\delta O F u$ et $(\square u, u)=(* O F u, * O F u)$, qui démontrent le 
THÉoRème. Pour qu'un vecteur $u$ soit analytique contrevariant, il faut ct il suffit que $\square u=0$. (Voir K. Yano [3]).

§3. Considérons un espace de Kähler-Einstein. Un tel espace est caractérisé par l'équation $K_{i}^{h}=k A_{i}^{h}, k$ étant une constante. L'opérateur $\Delta$ appliqué à un vecteur $u$ prend la forme $\Delta u:-\left(g^{j i} \nabla_{j} \nabla_{i} u_{h}-k u_{h}\right)$ et $\square$ appliqué à un vecteur $u$ la forme $\square u:-\left(g^{j i} \nabla_{j} \nabla_{i} u^{h}+k u^{h}\right)$ et par conséquent on a $\left.\square u=\right\lrcorner u$ $-2 k u$, d'où

ThÉoRÈme. Pour qu'un vecteur dans un espace compact de Kähler-Einstein soit analytique contrevariant, il faut et il suffit que $\Delta u=2 \mathrm{ku}$.

D'après Bochner [4], si $k>0$, il n'existe pas un vecteur analytique covariant et si $k<0$, il n'existe pas un vecteur analytique contrevariant. Si $k=0$, un vecteur analytique covariant ou contrevariant est parallèle.

Supposons donc que $k>0$ et prenons un vecteur analytique contrevariant $u$, alors on a $\Delta u=2 k u$, d'oì $\Delta d \grave{u} u=2 k d \delta u$, ce qui montre que $u^{\prime}=d o ̀ u$ est un vecteur analytique contrevariant. Or le vecteur $v=u-\frac{1}{2 k} w^{\prime}$ satisfait is $d v^{\prime}$ $=2 k v$, il est donc aussi un vecteur analytique contrevariant. Or $\delta v=\delta u$ $\left.-\frac{1}{2 k} \delta v^{\prime}=\delta u-\frac{1}{2 k} \delta d \delta u=\delta u-\frac{1}{2 k} \delta\right\lrcorner u=\delta u-o u=0$. Donc, le vecteur $v$, satisfaisant à $\Delta v=2 k v$ et $\delta v=0$, est un vecteur de Killing. Donc, on a démontré que $u=v+\frac{1}{2 k} v^{\prime}$ où $v$ est un vecteur de Killing et $w^{\prime}$ un vecteur analytique contrevariant.

Il est facil de voir que si $w^{\prime}$ est un vecteur analytique contrevariant, alors $F w^{\prime}: F_{i}^{h} w^{\prime i}$ l'est aussi. D'autre part, $u^{\prime}$ étant un vecteur gradient, on a $\partial\left(F\left(u^{\prime}\right)\right.$ $=\nabla_{j} F^{j i} w_{i}^{\prime}=F^{j i} r_{j} w_{i}^{\prime}=0$, d'où, le vecteur $w=-\frac{1}{2 k} F w^{\prime}$ est un vecteur de Killing. Le vecteur $\frac{1}{2 k} w^{\prime}$ étant de la forme $\frac{1}{2 k} w^{\prime}=F w$, on a $u=v+F u$ où $v$ et $u^{\prime}$ sont tous les deux les vecteurs de Killing.

Si le vecteur analytique contrevariant $u$ admet deux telles décompositions $u=v_{1}+F u_{1}$ et $u=v_{2}+F w_{2}$, on en tire $\left(v_{1}-v_{2}\right)+F\left(w_{1}-w_{2}\right)=0$, d'où, $v_{1}-v_{2}$ étant un vecteur de Killing, $\delta F\left(u_{1}-u_{2}\right)=0$, qui montre que $F\left(u_{1}-u_{2}\right)$, étant gradient et ayant la codifférentielle nulle, est nul, d'où $w_{1}^{\prime}=w_{2}$ et par conséquent $v_{1}=v_{2}$, ce qui démontre le théorème de M. Matsushima.

$\S 4$. Soit $u$ un vecteur analytique contrevariant: $\Delta u=2 k u$ et $u=h+d a+o b$ la décomposition de de Rham-Kodaira [2] de $u, h$ étant un vecteur harmonique. 
On a $\Delta u=\Delta(h+d a+\delta b)=2 k(h+d a+\delta b)$, d'où $-2 k h+d(\Delta a-2 k a)$ $+\delta(\Delta b-2 k b)=0$, par conséquent, si $k \neq 0$, on a $h=0, \Delta d a=2 k d a, \Delta \delta b=2 k \delta b$. Donc $d a$ et $\delta b$ sont tous les deux vecteurs analytiques contrevariants. Si l'on pose $d a=w^{\prime}, \delta b=v$, on a $\delta v=0, \quad \delta\left(-F w^{\prime}\right)=0$, donc $v$ et $-F w^{\prime}=w$ sont tous les deux les vecteurs de Killing et on a $u=v+F w$. L'unicité de cette décomposition se démontre de la même manière que la précédente.

\section{Bibliographie}

[1] Y. Matsushima: Sur la structure du groupe d'homéomorphismes analytiques d'une certaine variété kählérienne. Nagoya Mathematical Journal, 11 (1957), 145-150.

[2] G. de Rham et K. Kodaira: Harmonic integrals. Institute for Advanced Study (1950).

[3] K. Yano: Some integral formulas and their applications. To appear in Michigan Journal of Mathematics.

[4] K. Yano et S. Bochner : Curvature and Betti numbers. Annals of Mathematics Studies, No. 32 (1953).

Université de Tokyo 